\title{
Changes in diagnosis rates and behavioural traits of autism spectrum disorder over time
}

\author{
Ginny Russell, Stephan Collishaw, Jean Golding, Susan E Kelly and Tamsin Ford
}

\section{Background}

The increased proportion of UK children diagnosed with autism spectrum disorder (ASD) has been attributed to improved identification, rather than true increase in incidence.

\section{Aim}

To explore whether the proportion of children with diagnosis of ASD and/or the proportion with associated behavioural traits had increased over a 10-year period.

\section{Method}

A cross-cohort comparison using regression to compare prevalence of diagnosis and behavioural traits over time. Participants were children aged 7 years assessed in 1998/1999 $(n=8139)$ and 2007/2008 ( $n=13831)$.

\section{Results}

During 1998/1999, 1.09\% (95\% Cl 0.86-1.37) of children were reported as having ASD diagnosis compared with $1.68 \%$ (95\% Cl 1.42-2.00) in 2007/2008: risk ratio (RR) $=1.55$
(95\% Cl 1.17-2.06), $P=0.003$. The proportion of children in the population with behavioural traits associated with ASD was also larger in the later cohort: $\mathrm{RR}=1.61(95 \% \mathrm{Cl} 1.35-1.92), P<0.001$. Increased odds of diagnosis at the later time point was partially accounted for by adjusting for the increased proportion of children with ASD-type traits.

\section{Conclusions}

Increased ASD diagnosis may partially reflect increase in rates of behaviour associated with ASD and/or greater parent/ teacher recognition of associated behaviours.

\section{Declaration of interest}

None.

\section{Copyright and usage}

(C) The Royal College of Psychiatrists 2015. This is an open access article distributed under the terms of the Creative Commons Non-Commercial, No Derivatives (CC BY-NC-ND) licence.
The number of children diagnosed with autism spectrum conditions over the past 40 years has increased substantially. ${ }^{1}$ When the first UK prevalence study was conducted, autism was thought to be a rare condition that occurred in 1 child per $2500 .^{2}$ Today, estimates suggest at least 1 in 100 children have an autism spectrum disorder (ASD). ${ }^{3}$ Latest US parent report estimates are that 1 in 68 children (1.5\%) are diagnosed with ASD by the age of $8,{ }^{4}$ although the trend for increasing diagnosis may be levelling off in the UK. ${ }^{5}$ The reasons behind the rising rate of diagnosis of ASD are hotly debated. The crux of the debate is whether increases are entirely an artefact of changing diagnostic practice, greater awareness and different methodologies used to estimate prevalence, or whether there is a true increase in the incidence, i.e. there is an increase in the number of children with behaviours that underlie autism. Revisions to diagnostic criteria certainly provide a partial explanation. For example, the inclusion of Asperger syndrome in DSM-IV in 1994 meant that more children were identified in the ASD category. Moreover, there is greater awareness of the condition both at home and in the clinic, ${ }^{6}$ particularly after media exposure and the work of ASD charities. It has been argued there is no 'epidemic' of autism and that apparent rises in prevalence can be attributed to the combination of children with milder difficulties receiving the diagnosis due to broadened diagnostic criteria, greater public and clinical awareness and improved case identification including diagnosis of children with typical attainment. ${ }^{7}$ However, many people affected by these conditions, particularly parents, clinicians and educators believe that as well as changes in diagnostic practice and increasing awareness there is additionally a true rise in the underlying incidence of ASD. ${ }^{8}$ As the DSM-5 puts it: 'it remains unclear whether higher rates reflect an expansion of the diagnostic criteria of DSM-IV to include subthreshold cases, increased awareness, differences in study methodology or a true increase in the frequency of autism spectrum disorder'.
Our objectives were therefore (a) to confirm an increase in parent-reported ASD diagnosis at age 7 in the UK between two time points (two population-based cohorts from 1998/1999 and 2007/2008), (b) to compare the proportions of children with behaviours associated with autism over the same time period, and (c) to reassess the increase in rate of diagnosis of ASD after accounting for any change in the proportion of children with behaviour associated with autism. In light of recent discussions about the under-identification of ASD in females ${ }^{10}$ and the diagnosis of younger children, ${ }^{7}$ a secondary aim was to check whether any increase in diagnosis over time was proportionally the same for females and younger children.

\section{Method}

The study used a cross-cohort comparison design utilising 'likefor-like' closely similar or identical measures related to ASD-type behaviour and repeated across two unselected population cohorts. The like-for-like approach aims to utilise the same tools of measurement in two or more population-based birth cohorts in which children are born at different times. The aim is assess time trends: ideally constructs of interest should be measured by exactly the same instruments in each cohort, and administered in the same way, or as near as is possible. The primary advantage of this approach is that 'like-for-like' comparisons extend to the whole population rather than focusing on selected subgroups seen in clinical practice. ${ }^{11}$ A primary limitation is that comparable measures relate to symptoms and behaviours assessed using instruments included in national cohort studies rather than validated assessments of ASD. In this study, we compared two UK birth cohorts in cross-section when children were age 7 or 8 years old across a 10-year period (1998/1999-2007/2008). A recent cross-national comparison of six successive birth cohorts in Denmark, Australia, Finland and Sweden found rates of ASD 


\begin{tabular}{|c|c|c|}
\hline \multicolumn{3}{|c|}{ ALSPAC child age 9 years } \\
\hline \multicolumn{3}{|c|}{$\begin{array}{l}\text { 'Have you ever been told that your child has } \\
\text { autism, Asperger syndrome or autism } \\
\text { spectrum disorder?' }\end{array}$} \\
\hline Response & Coded as & $n$ \\
\hline Yes & $\begin{array}{c}\text { ASD } \\
\text { diagnosis }\end{array}$ & 96 \\
\hline No & $\begin{array}{c}\text { No ASD } \\
\text { diagnosis }\end{array}$ & 7688 \\
\hline No response & $\begin{array}{c}\text { No ASD } \\
\text { diagnosis }\end{array}$ & 437 \\
\hline Not completed & Missing & 6903 \\
\hline Supplementary & stion: 'age a & nosis?' \\
\hline Response & Coded as & $n$ \\
\hline Age 8,9 & $\begin{array}{l}\text { Excluded } \\
\text { from ASD } \\
\text { diagnosis }\end{array}$ & 13 \\
\hline
\end{tabular}

Proportion with parent-reported ASD diagnosis at 7 years -1998

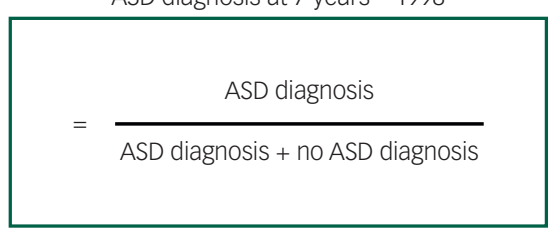

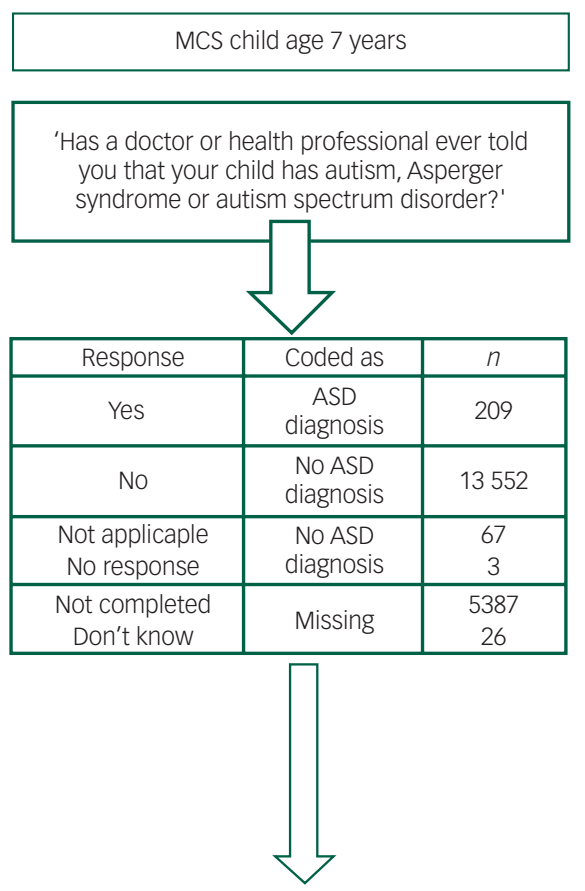

Proportion with parent-reported ASD diagnosis at 7 years -2008

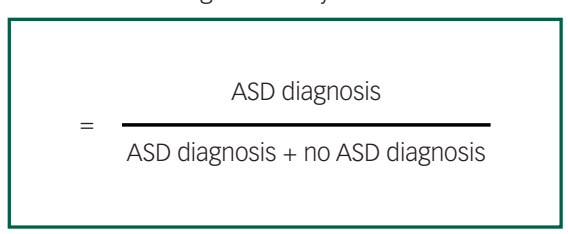

Fig. 1 Steps in deriving parent-reported autism spectrum disorder (ASD) diagnosis at age 7 in both cohorts.

ALSPAC, Avon Longitudinal Study of Parents and Children; MCS, Millennium Cohort Study.

diagnosis increased in children aged 7 from 1997/1999 through $2006 / 2008$ in all four countries. ${ }^{12}$

\section{Samples}

The samples were two UK birth cohorts: the Avon Longitudinal Study of Parents and Children (ALSPAC) and the Millennium Cohort Study (MCS). In ALSPAC, 99\% of children were age 7 in 1998 or 1999; in MCS $99 \%$ of children reached this age in 2007 or 2008. According to our review of UK cohort studies, these are the only two recent studies that include information about clinical diagnosis of ASD and similar measures of behaviour associated with ASD.

The ALSPAC cohort is a whole population birth cohort study of pregnant women and their children in a defined geographical area of the UK. ALSPAC prospectively examines influences on health and development across the life-course. All pregnant women resident in the Avon region of South West England were eligible for inclusion (just over 20000 ) and 14541 were initially recruited, which resulted in 14062 live births in 1991-1992. When children were 7 years old, 706 additional eligible families were recruited. Details of the measures taken throughout their childhood and adolescence have been tabulated elsewhere. ${ }^{13}$ Social and demographic features of the ALSPAC cohort were broadly representative of the overall population in the UK as measured by the 1991 UK national census. ${ }^{14}$ The ALSPAC study website contains details of all the data that are available through a fully searchable online data dictionary and gives further details of representativeness of the sample.
The MCS is a UK-representative birth cohort study that used a stratified cluster sampling design to examine health and developmental outcomes across the life-course of its study children. Children born between 2000 and 2002 and listed on the Child Benefit Records, a database registering more than $98 \%$ of children in the UK, were eligible. Study design was stratified by UK country (England, Scotland, Wales and Northern Ireland), and further stratified by ethnic group and level of social disadvantage; details of the sampling design are documented by the curators. ${ }^{16,17}$ Data have been collected since children were 9 months old. About 19519 families were initially recruited to the MCS cohort, and parent report of ASD diagnosis was recorded at age 7. Data are freely available to accredited researchers via the UK data service website (ukdataservice.ac.uk).

Ethical approval for the ALSPAC study was obtained from the ALSPAC Law and Ethics Committee. For MCS, written informed consent was obtained from parents at each data collection stage for their participation and the participation of their child; further details are provided in the MCS ethical review. ${ }^{15}$ Analyses were conducted anonymously, with researchers having no access to participant identities. Additional ethical approval for the analyses reported in this study was granted by the University of Exeter Medical School Ethics committee.

\section{Measures}

\section{Parent-reported ASD diagnosis}

ASD diagnosis was reported by parents in both cohorts. Figure 1 shows the exact wording of the question presented to the parents 
and the response categories. 'Autism spectrum disorder' is adopted throughout this article to describe autism spectrum conditions as this was the term used in the MCS questionnaire (Fig. 1). Parent-reported data on ASD diagnosis were collected using a self-completion questionnaire in ALSPAC and recorded during face-to-face interviews in the MCS. This parent-reported question has been repeatedly used to estimate rates of diagnosis of ASD in the USA. ${ }^{16}$ The sample eligible for this analysis was limited to those children whose parents reported on ASD diagnosis status. In addition, the analyses included only one child per family (second born twin siblings were excluded) as ASD traits are likely to be co-inherited. ${ }^{17}$ The included study sample size was 8139 in ALSPAC (60\% of total sample) and 13831 in MCS (70\% of total sample). Selective attrition was dealt with using weightings (see Analysis section). Table 1 shows the distribution of early predictors of (a) the complete early sample, (b) the unweighted analysis sample and (c) the analysis sample (weighted).

To validate parent-reported diagnosis of ASD, parent report of an autism diagnosis was checked against the medical report of clinical ASD diagnosis (which was only available in ALSPAC). Clinical ASD diagnosis was obtained from medical records. ${ }^{18}$ Briefly, individuals in the ALSPAC cohort who had a diagnosis relating to any form of developmental delay or listed as special educational needs were recorded from the computer systems of relevant National Health Service trusts. The records were matched against the ALSPAC cohort to confirm that the child was a member of the study and that permission had been given to search their health records. A team of three experienced researchers then searched hospital medical records (in-patient and out-patient) and community child health records (including child development team records) to identify children who had a diagnosis of ASD made after a multidisciplinary assessment. No direct contact was made with any of the children, parents or clinicians. The sensitivity of parent report in identifying medical diagnosis was $95 \%$ and the specificity was $99 \%$, indicating it was a good but not perfect indicator of clinical diagnosis (online supplement Table DS1).

\section{Behaviours associated with autism}

Data on behaviours associated with autism spectrum traits occurring in multiple settings were collected in both cohorts via teacher assessments and by parent report. Online supplement Table DS2 shows how measures tapped into the three domains of impairment in social skills, impairment in communication and adherence to routines listed in ICD-10 ASD criteria, ${ }^{19}$ and the individual items used, and how they were scored.

Parent- and teacher-rated subscales from the Strengths and Difficulties Questionnaire (SDQ) of prosocial behaviour, peer problems, and 'difficulty coping with new situations' ${ }^{20}$ were recorded in both cohorts. Answers to SDQ reports were given on the basis of the child's behaviour over the past 6 months or in the concurrent school year. We also utilised foundation stage assessments of social development and language for communication made by teachers in children's first year of school ${ }^{21}$ which were also included in both cohorts. Distributions of foundation stage assessment scores of communication and social development from educational data in each cohort are given in online supplement Fig. DS1.

\section{The composite autism-type trait score}

Given that autism is conceptualised as the constellation of difficulties across multiple domains of impairment we used the eight measures described above to derive a composite autism-type trait score (CATS). First, data were pooled from both cohorts and each of the eight indicator measures was standardised $(M=0$, s.d.=1). Some were reverse-coded so a higher score indicated greater impairment. CATS comprised the overall mean score across each of component indicator measures (calculated where data were available for a minimum of five indicator scales). This resulted in 4530 children with CATS scores in ALSPAC (56\% of the age 7 sample) and 11210 in MCS (81\% of the age 7 sample). The CATS score had a positive skew-normal distribution. Children with ASD diagnosis mostly appeared at the skewed tail end (Fig. 2). This method was akin to that initiated in previous work ${ }^{22}$ but in order to allow cross-cohort comparisons we used an amended approach that only incorporated autism-related behaviours recorded in both cohorts.

To test whether the same proportion of children had autismrelated behaviours in $2007 / 2008$ as $1998 / 1999$, we specified a cutoff (top 5\% of CATS score based on scores pooled across both cohorts). The top 5\% range of CATS was selected as it captured $67 \%$ of children with a diagnosis of ASD, and the specificity (the

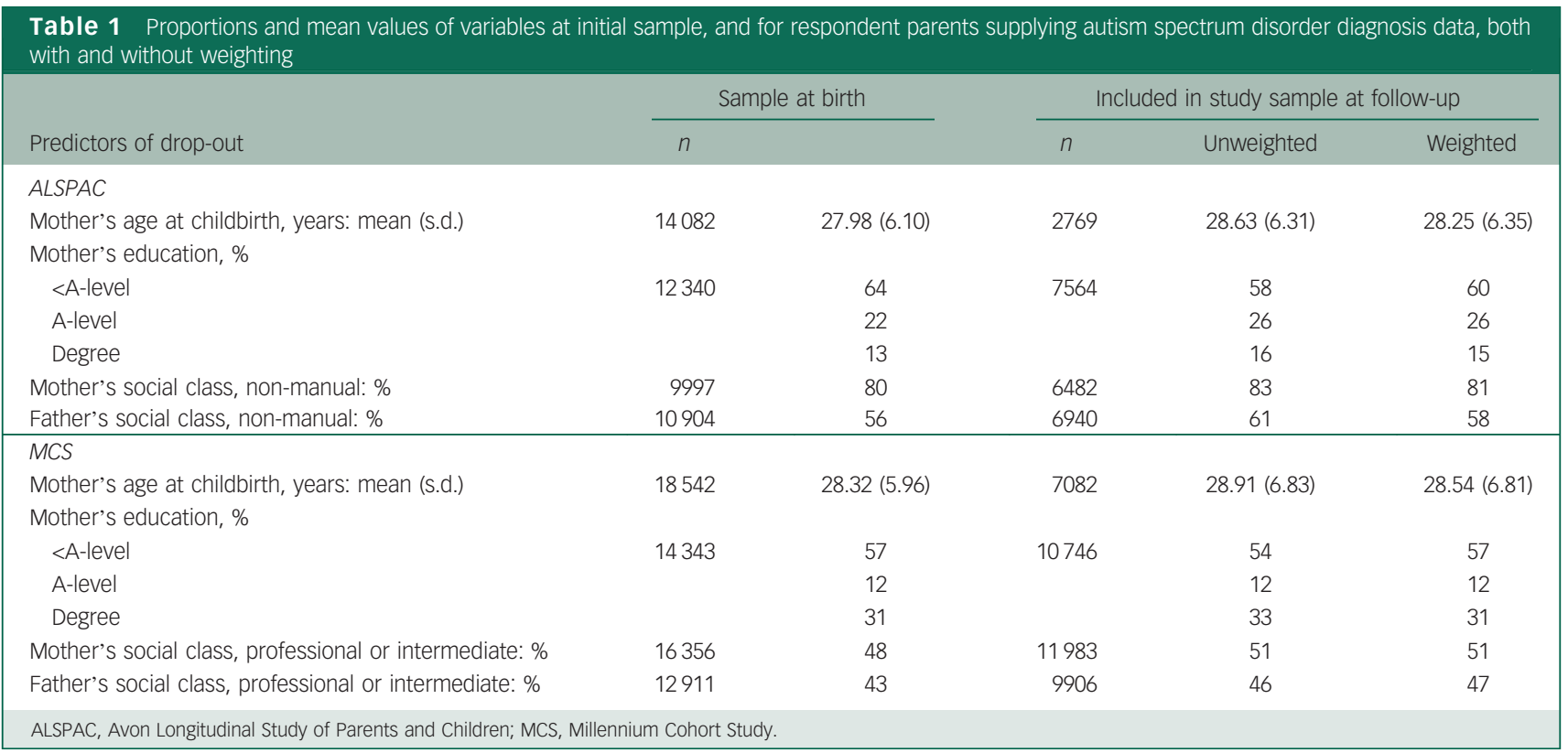




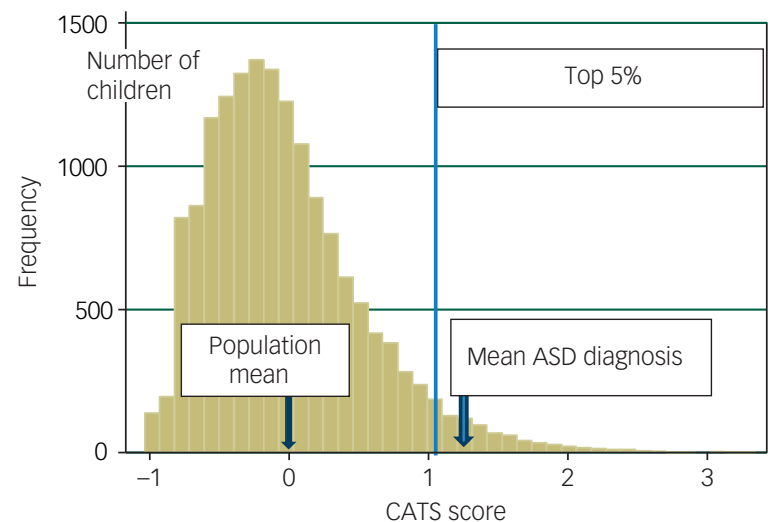

Fig. 2 Distribution of composite autism-type trait score (CATS) in both cohorts together $n=15750$. ASD, autism spectrum disorder

percentage of children who were correctly identified as not having the condition) was 95\% (see online supplement Table DS3 for cross-tabulation). Figure 2 illustrates the cut-off point in the pooled study samples. A child above this cut-point likely had poor communication, was less able to sustain peer relationships, and/or was afraid of new situations and/or did not share easily or empathise well. The category was labelled 'high CATS'.

\section{Statistical analysis}

First, the unadjusted association between individual behavioural subscales and ASD diagnosis was checked in each cohort. We predicted that these behaviours would be strongly associated with the parent report of ASD diagnosis, and the unadjusted association was carried out to check this was the case. Data were pooled, then the percentage of children with a parent-reported ASD diagnosis and the percentage that had high CATS behaviour above cut-off were obtained for each time point (1998 or 2008) with $95 \%$ confidence intervals. Risk ratios (RRs) and odds ratios (ORs) with confidence intervals are presented to quantify the prevalence of diagnosis and high CATS status in the MCS cohort (reported in 2007/2008) relative to the ALSPAC cohort (reported in 1998/ 1999). Logistic regression models were fitted to estimate ORs using the logit function, and RRs were estimated with generalised linear modelling using a combination approach. These were models with high CATS status or ASD diagnosis as the outcome and cohort (time point) as the predictor.

The revised OR for ASD diagnosis in the MCS cohort relative to the ALSPAC cohort was then re-estimated adjusting for both continuous CATS scores and high CATS (top 5\%) status. If there was a true underlying increase in children with ASD then we would expect there to be an increase in the number of children with impaired (top 5\%) CATS. Furthermore, we would expect that accounting for increase in children high CATS would partially or fully explain increased risk of diagnosis in the later cohort.

MCS supplies weightings to make the sample representative of the UK population as a whole at age 7: these take into account the stratified sample, over recruitment of subgroups, and attrition. To account for attrition in ALSPAC, non-response weights were derived based on prior predictors of attrition available for the whole cohort at birth (maternal education, maternal and paternal social class and maternal age at child's birth) as in previous crosscohort comparisons. ${ }^{23}$ Table 1 characterises missing data and shows the efficacy of weights in addressing selective non-response. Weights shifted the distribution so that it was more representative of the sample at birth but did not completely correct it. For example, the adjustment did not seem to fully correct for the selective drop-out of mothers of lower education.

A secondary objective was to characterise how children diagnosed with ASD differed between 1998/1999 and 2007/2008 in terms of gender and age of diagnosis. Logistic regression was carried out in the subsample of children with ASD diagnosis ( $n=291)$. Cohort (time point) was the predictor and child gender or age of diagnosis was the outcome. This was to establish whether the later time point predicted whether females and/or younger children were more likely to receive the ASD diagnosis.

All analyses were conducted using Stata 13 software. Descriptive statistics were generated using weightings and for MCS the UK sampling frame (svy: command in Stata). The ORs generated were derived in three ways in order to check that estimates were reliable - given that MCS had an integral sampling frame and ALSPAC did not. The ORs were initially derived with and without MCS survey frame: (a) inserting ALSPAC data into the MCS survey frame, (b) using attrition weightings only without any sampling frame and (c) OR estimates were calculated manually from descriptive statistics in MCS generated using sampling frame and weightings, and using derived weightings for ALSPAC. No discrepancies in results were detected using these three divergent methods, despite the use of different survey frames in the two cohorts. We therefore concluded estimates of ORs were reliable. Adjusted analyses were subsequently carried out using the second method.

\section{Results}

All behaviour scales were strongly associated with ASD diagnosis in each cohort (online supplement Table DS4).

In the 1998 ALSPAC cohort, $1.09 \%$ of children were reported to have had an ASD diagnosis (95\% CI 0.86-1.37). In the 2008 MCS cohort, $1.68 \%$ children were reported as having an ASD diagnosis (95\% CI 1.42-2.00). Thus odds of a child having an ASD diagnosis increased in the later cohort: $\mathrm{OR}=1.56$ (95\% CI 1.16-2.08); $P=0.003$. The corresponding increased risk (RR) of being diagnosed with ASD in the later cohort was 1.55 (95\% CI 1.17-2.06), $P=0.003$.

In the earlier 1998/1999 cohort, $4.26 \%$ (95\% CI 3.64-4.98) of children fell in the high CATS category; whereas in the 2007/2008 cohort, 6.86\% (95\% CI 6.29-7.49) had high CATS traits (top 5\%). There was strong evidence to suggest the risk/odds of having traits in the top $5 \%$ of the CATS score were greater in the later cohort, $\mathrm{OR}=1.65 \quad(95 \% \quad \mathrm{CI} \quad 1.38-1.99) / \mathrm{RR}=1.61 \quad(95 \%$ CI $\quad 1.35-1.92)$, $P<0.001$.

Effect size for increased odds of diagnosis in the later cohort (MCS) relative to the earlier cohort (ALSPAC) was reduced and became non-significant at $5 \%$ level after adjusting for CATS score: $\mathrm{OR}=1.29,95 \%$ CI $0.94-1.97, P=0.22$, or for high CATS status $\mathrm{OR}=1.17,95 \% \mathrm{CI} 0.80-1.72, P=0.41$. Together, the adjusted results suggest an increased number of children with behaviours associated with autism, could be responsible, in part, for the increase in parent-reported ASD diagnosis observed between the two cohorts.

Table 2 illustrates how the characteristics of the diagnosed samples varied between cohorts. The proportion of boys with ASD diagnosis was higher than the proportion of girls in both cohorts, consistent with well-established gender ratios for ASD. No differences were detected in gender ratios of diagnosed children between cohorts, suggesting the increased application of the diagnosis was similar for both genders, and there was no detectable difference in age of diagnosis. 
Table 2 Characteristics of children with parent-reported ASD diagnosis ( $n=291)$ by cohor

\begin{tabular}{|c|c|c|c|c|}
\hline & $\begin{array}{c}\text { ALSPAC } \\
1997 / 1998 \\
\text { Total } n=82\end{array}$ & $\begin{array}{c}\text { MCS } \\
2007 / 2008 \\
\text { Total } n=209\end{array}$ & $\begin{array}{c}\text { Test of difference } \\
\text { OR }(95 \% \mathrm{Cl})\end{array}$ & $P$ \\
\hline Female, \% & 26 & 18 & $0.95(0.89-1.01)$ & 0.12 \\
\hline Age at diagnosis ${ }^{a}$ early ( $<5$ years), \% & 72 & 60 & $0.56(0.27-1.18)$ & 0.13 \\
\hline
\end{tabular}

\section{Discussion}

In line with other evidence from the UK, our findings confirm that the report of an ASD diagnosis by parents was higher in MCS in 2007/2008 than in ALSPAC in 1998/1999. Other UK studies based on ASD diagnosis recorded in general practice have shown stark increases from 1988 to 2001, but suggest the trend may have levelled off more recently. ${ }^{5}$ Lower thresholds for diagnosis in successive revision to diagnostic criteria meant more children were included in the ASD category throughout the time period in question, though a meta-analysis suggests the most recent changes to the diagnostic classification system in DSM-5 could result in a decrease in the number of children diagnosed in future. ${ }^{24}$ However, our main finding implies that increasing rates of ASD diagnosis in the UK over this period may not be solely an artefact of changing diagnostic practice and clinical recognition, but there may be an increase in the proportion of children with associated behaviours. Our data show, according to parent report, children were over 1.5 times as likely to be diagnosed with ASD in 2007/ 2008 compared with $1998 / 1999$. The rest of the results shed light as to why this may have occurred.

The proportion of children with autism-related behaviours was greater in the later cohort. Adjusting for increases in autism-related traits led to a decline in the relative odds of being diagnosed with ASD in the later cohort, with the relationship becoming nonsignificant. This suggests that the observed increases in number of children with behaviours associated with autism partially accounted for the increased diagnosis rates. The finding chimes with experience of teachers: a UK survey found $67 \%$ thought there were more children with ASD in 2000 than $1995 .{ }^{25}$ Parents have also suggested rates of autism-related behaviour are greater than before. ${ }^{8}$

A recent study of a population-based cohort of over 2 million children estimated that substantially more of the risk of ASD is conferred by environmental factors than previously thought. ${ }^{26}$ It is conceivable that novel environmental factors such as social changes with biological implications, for example, couples having children later could influence trends in population prevalence of ASD. ${ }^{27}$ A recent review of time trends in child and adolescent mental health ${ }^{11}$ has highlighted that even where diagnostic criteria and practice have changed, there may still be strong evidence for real additional change in population prevalence (e.g. with respect to adolescent depression and anxiety).

Nevertheless, caution is needed in interpreting the findings. 'Reporting drift' is the process through which parent and teacher reporting of behaviours and impairment may change across time. For example, there may be more societal emphasis on impaired social skills and problems with communication at the later time point. A difficulty in the interpretation of time trend findings is the separation of true changes from reporting drift (even in general population cross-cohort comparisons such as this where other methodological artefacts such as changes in diagnostic practice are controlled). How do we know what is a facet of change in symptoms over time, and what is an artefact of changing standards of reporting? The current study used a variety of sources, parent report, teacher report, and school assessments. Such a variety of sources is acknowledged to be more likely to give a 'true' picture of underlying behaviours. However, reporting drift cannot be ruled out as parents and teachers may still have systematically over-identified autism-type difficulties in the later cohort.

\section{Strengths and limitations}

The two cohorts, although both population-based, had differing geographical reach and sampling frames. The MCS was representative of the UK population as a whole. The ALSPAC survey, despite being a regional study was broadly representative of the UK population at the 1991 census in most aspects of social demography ${ }^{14}$ but differences in ASD diagnostic practice by region cannot be discounted. There was a lack of geographical variation in ASD diagnosis in MCS, albeit by country not county, but data from the USA suggest that there may be variations in ASD diagnosis across regions ${ }^{28}$ that we could not account for. The study's strengths include the use of population-based samples, where non-response weights were applied to account for known predictors of attrition, with no evidence for differential selective non-response between cohorts. The measures used were closely similar, although they were not always administered and recorded in an identical way. For example, the ASD diagnosis question was worded differently (Fig. 1) and asked via postal questionnaire in the ALSPAC cohort and via face-to-face questionnaire for the MCS cohort. Replication of the findings is therefore important.

Our findings would have been more relevant to the ASD prevalence question whether well-validated autism-specific measures (e.g. the Autism Diagnostic Observation Schedule) were used, but no such measures are presently available across UK population cohorts. The trait scores for the two cohorts were used to generate a CATS scale with reasonable sensitivity and specificity, making it a suitable, although limited, indicator of some ASD traits. We are not claiming that the CATS scale represented 'autism' but a child with high CATS' tail likely had poor communication, was less able to sustain peer relationships, and/or was afraid of new situations, and/or did not share easily or empathise well. However, none of the items asked about restricted interests, repetitive behaviours, or stereotypic behaviours, which represent a key facet of the ASD phenotype. High CATS was used to characterise behaviours associated with ASD but is not a validated ASD measure. The CATS scale simply maximised the data available in a way that optimised our ability to answer the study questions, which the individual scales could not.

At present, like-for-like comparisons of unselected cohorts are lacking with respect to ASD. A Scandinavian team did assess the time-trend in Sweden recently, but claim to have found no evidence of increasing numbers of children displaying the ASD phenotype over time. ${ }^{29}$ However these authors did report a significant increase in mean scores for the autism phenotype over time but fail to elaborate in their discussion. We believe that our results indicate the debate over increasing prevalence of ASD is ongoing; increasing rates of ASD diagnosis in the UK in the past 
may not be solely due to changes in diagnostic criteria and clinical practice. Our findings may reflect a true increase in population traits and behaviours associated with ASD. Alternatively, shifts in parent and teacher recognition and reporting of such traits may explain the findings. Further research is needed to distinguish these two possibilities.

Ginny Russell, PhD, Institute of Health Research, University of Exeter Medical School, Exeter, UK: Stephan Collishaw, PhD, Institute of Psychological Medicine and Clinical Neurosciences, Cardiff University School of Medicine, Cardiff, UK; Jean Golding, Centre for Child \& Adolescent Health, School of Social \& Community Medicine, University of Bristol, Bristol, UK; Susan E. Kelly, Department of Sociology, Philosophy and Anthropology, School of Social Science, University of Exeter, Exeter, UK; Tamsin Ford, Institute of Health Research, University of Exeter Medical School, Exeter, UK

Correspondence: Ginny Russell, Institute of Health Research, University of Exeter Medical school, South Cloisters, St Luke's Campus, Exeter EX1 2LU, UK. Email: g.russell@ex.ac.uk

First received 15 May 2015, final revision 4 Aug 2015, accepted 19 Aug 2015

\section{Funding}

The research was specifically funded by the ESRC Secondary Data Analysis Initiative, Grant Number ES/K003356/1.

\section{Acknowledgements}

We are extremely grateful to all the families who took part in this study, the midwives for their help in recruiting them, and the ALSPAC team, which includes interviewers, compute and laboratory technicians, clerical workers, research scientists, volunteers, managers, receptionists and nurses. The UK Medical Research Council (Grant ref: 74882), the Wellcome Trust (Grant ref: 076467) and the University of Bristol currently provide core support for ALSPAC. We thank the Millennium Cohort Study families for their time and cooperation, as well as the Millennium Cohort Study team at the Institute of Education, London, UK.

\section{References}

1 Weintraub K. The prevalence puzzle: autism counts. Nat News 2011; 479: 22-4.

2 Lotter V. Epidemiology of autistic conditions in young children. Soc Psychiatry 1966; 1: 124-35.

3 Baron-Cohen S, Scott FJ, Allison C, Williams J, Bolton P, Matthews FE, et al. Prevalence of autism-spectrum conditions: UK school-based population study. Br J Psychiatry 2009; 194: 500-9.

4 The Centers for Disease Control and Prevention. Prevalence of Autism Spectrum Disorder among Children Aged 8 Years - Autism and Developmental Disabilities Monitoring Network, 11 Sites, United States, 2010. Morbidity and Mortality Weekly Report, 2014

5 Taylor B, Jick H, MacLaughlin D. Prevalence and incidence rates of autism in the UK: time trend from 2004-2010 in children aged 8 years. BMJ Open 2013; 3 e003219.

6 Silverman C. Understanding Autism: Parents, Doctors, and the History of a Disorder. Princeton University Press, 2011.

7 Fombonne E. Is there an epidemic of autism? Pediatrics 2001; 107: 411-2.

8 Russell G, Kelly S, Golding J. A qualitative analysis of lay beliefs about the aetiology and prevalence of autistic spectrum disorders. Child Care Health Dev 2010; 36 431-6.
9 American Psychiatric Association. Diagnostic and Statistical Manual of Mental Disorders, Fifth Edition (DSM-5). APA, 2013

10 Holtmann M, Bölte S, Poustka F. Autism spectrum disorders: sex differences in autistic behaviour domains and coexisting psychopathology. Dev Med Child Neurol 2007; 49: 361-6.

11 Collishaw S. Annual research review: secular trends in child and adolescent mental health. J Child Psychol Psychiatry 2015; 56: 370-93.

12 Atladottir HO, Gyllenberg D, Langridge A, Sandin S, Hansen SN, Leonard H, et al. The increasing prevalence of reported diagnoses of childhood psychiatric disorders: a descriptive multinational comparison. Eur Child Adolesc Psychiatry 2015; 24: 173-83.

13 Boyd A, Golding J, Macleod J, Lawlor DA, Fraser A, Henderson J, et al. Cohort Profile: the 'Children of the 90s'-the index offspring of the Avon Longitudinal Study of Parents and Children. Int J Epidemiol 2013; 42: 111-27.

14 Golding J, Pembrey M, Jones R. ALSPAC - the Avon Longitudinal Study of Parents and Children. I. Study methodology. Paediatr Perinat Epidemiol 2001; 15: 74-87.

15 Shepherd P. Millennium Cohort Study: Ethical Review and Consent. Centre for Longitudinal Studies, Institute of Education, 2012

16 Boyle CA, Boulet S, Schieve LA, Cohen RA, Blumberg SJ, Yeargin-Allsopp M, et al. Trends in the prevalence of developmental disabilities in US children, 1997-2008. Pediatrics 2011: 127: 1034-42.

17 Constantino JN, Todd RD. Autistic traits in the general population: a twin study. Arch Gen Psychiatry 2003; 60: 524-30.

18 Williams $\mathrm{E}$, Thomas $\mathrm{K}$, Sidebotham $\mathrm{H}$, Emond A. Prevalence and characteristics of autistic spectrum disorders in the ALSPAC cohort. Dev Med Child Neurol 2008; 50: 672-7.

19 World Health Organization. International Classification of Diseases and Related Health Problems (ICD-10) [Internet]. WHO, 1992.

20 Goodman R. Psychometric properties of the strengths and difficulties questionnaire. J Am Acad Child Adolesc Psychiatry 2001; 40: 1337-45.

21 Department for Education and Skills. Foundation Stage Profile: Handbook. QCA Publications, 2003

22 Steer CD, Golding J, Bolton PF. Traits contributing to the autistic spectrum. PLOS One 2010; 5: e12633.

23 Collishaw S, Goodman R, Pickles A, Maughan B. Modelling the contribution of changes in family life to time trends in adolescent conduct problems. Soc Sci Med 2007: 65: 2576-87.

24 Barnard J, Potter D, Broach S, Prior A. Autism in Schools: Crisis or Challenge? National Autistic Society, 2000

25 Kulage KM, Smaldone AM, Cohn EG. How will DSM-5 affect autism diagnosis? A systematic literature review and meta-analysis. J Autism Dev Disord 2014; 44: 1918-32.

26 Sandin S, Lichtenstein P, Kuja-Halkola R, Larsson $\mathrm{H}$, Hultman CM, Reichenberg A. The familial risk of autism. JAMA 2014: 311: 1770-7.

27 Sandin S, Hultman CM, Kolevzon A, Gross R, MacCabe JH, Reichenberg A. Advancing maternal age is associated with increasing risk for autism: a review and meta-analysis. J Am Acad Child Adolesc Psychiatry 2012; 51: 477-86.e1

28 Liu K, King M, Bearman PS. Social influence and the autism epidemic. Am J Sociol 2010; 115: 1387-434.

29 Lundström S, Reichenberg A, Anckarsäter H, Lichtenstein P, Gillberg C. Autism phenotype versus registered diagnosis in Swedish children: prevalence trends over 10 years in general population samples. BMJ. 2015; 350: 1961 